\title{
Belphégor
}

\section{La forêt et ses habitants fantastiques dans les séries télévisées anglophones}

\section{François-Ronan Dubois}

\section{(2) OpenEdition}

\section{Journals}

Édition électronique

URL : http://journals.openedition.org/belphegor/457

DOI : 10.4000/belphegor.457

ISSN : 1499-7185

Éditeur

LPCM

Référence électronique

François-Ronan Dubois, «La forêt et ses habitants fantastiques dans les séries télévisées

anglophones », Belphégor [En ligne], 12-1 | 2014, mis en ligne le 15 juin 2014, consulté le 18 avril 2019. URL : http://journals.openedition.org/belphegor/457; DOI : 10.4000/belphegor.457

Ce document a été généré automatiquement le 18 avril 2019

\section{(c) (1) () $\Theta$}

Belphégor est mis à disposition selon les termes de la Licence Creative Commons Attribution - Pas d'Utilisation Commerciale - Pas de Modification 4.0 International. 


\title{
La forêt et ses habitants fantastiques dans les séries télévisées anglophones
}

\author{
François-Ronan Dubois
}

1 Lieu privilégié de l'imaginaire merveilleux, la forêt sert traditionnellement de cadre aux rencontres entre le héros et des créatures fantastiques, qui aident ou contrarient sa quête romanesque; que l'on songe aux aventures du héros Perceforest dans le roman du même nom, à la rencontre de Raymondin avec Mélusine dans La Noble Histoire de Lusignan ou aux tribulations de Perceval dans le Conte du Graal, l'on se rend compte que la forêt se superpose à la grande route où surgissent les aventures. Selon Corinne Saunders, la forêt médiévale passe du «classical setting of encounters between gods and mortals ${ }^{1}$ » au "romance setting of the quest, the idyll and the adventure ${ }^{2}$ " (Saunders, 114). Ces représentations ne sont certes pas surprenantes, puisqu'elles répondent à une réalité géographique de l'époque médiévale. Si la forêt est alors omniprésente dans l'espace et, par conséquent, dans l'imaginaire de cet espace, elle devient, au tournant entre le vingtième et le vingt-et-unième siècles, à l'âge de la série télévisée, un espace marginal, laissant place à une confrontation entre la civilisation et la sauvagerie, autrement dit entre la ville et ses landes industrielles.

2 Lorsque poursuivi par ses antagonistes ou les poursuivant lui-même, le héros de série télévisée abandonne le centre-ville et ses passants, ce n'est pas pour rejoindre un territoire naturel mais un nouveau paysage artificiel, structuré par la trace, mais non par la présence de l'humanité : les courses-poursuites de la série d'espionnage Alias (J. J. Abrams, 2001-2006) conduisent invariablement le spectateur dans des paysages industriels génériques (usines désaffectées, gares ferroviaires de triage, entrepôts), dont la fonction précise n'est pas toujours clairement intelligible, mais qui servent de cadre à la sauvagerie. De la même façon, des séries d'anticipation comme Bugs (Brian Eastman et Stuart Doughty, 1995-1999) filment des villes modernes entièrement dépeuplées pour susciter chez le téléspectateur un sentiment d'inquiétante étrangeté, fait de familiarité et de distance. 
3 Il faut que la série télévisée réactive un univers médiéval pour que l'opposition entre la nature et la ville redoublent parfaitement celle du sauvage et du civilisé. Ainsi les héros de Stargate SG-1 (Jonathan Glassner et Brad Wright, 1997-2007), en arrivant par la Porte des Étoiles sur une nouvelle planète, traversent-ils souvent une étendue boisée avant de parvenir à un village d'apparence médiévale, disposition souvent reprise dans la série australienne de science-fiction Farscape (Brian Henson et Rockne O'Bannon, 1999-2003). De la même façon, dans la série de western et de science-fiction Firefly (Joss Whedon, 2002), le paysage désertique s'oppose frontalement aux installations sommaires des colons et, dans Revolution (Eric Kripke, 2012, en production), c'est encore l'anéantissement technologique qui redonne une place de choix aux étendues naturelles. Mais ces oppositions naissent à la fois d'un imaginaire de l'espace et d'un imaginaire de l'histoire : c'est précisément parce que ces séries filment quelque chose comme une conquête de l'ouest ou un village médiéval que la dichotomie entre monde naturel et monde artificiel retrouve son caractère intuitif et que la continuité est paradoxalement immédiate entre le village et la nature qui l'entoure.

4 Bien plus souvent, la forêt est donc un territoire parfaitement étranger, qui contient intrinsèquement la possibilité de l'inhumain. C'est dans une forêt, par exemple, que s'organise une sinistre chasse à l'homme sportive dans Dollhouse (Joss Whedon, 2009-2010) ; c'est encore dans la forêt de Dathomir que vivent les servantes du Côté Obscur de la Force dans Star Wars: Clone Wars (Genndy Tartakovsky et Henry Gilroy, 2003-2005). On ne se rend dans la forêt qu'après s'être préparé et, tout méticuleux que soient les héros, leurs précautions ne sont jamais à la hauteur du défi qui les y attend.

\section{Buffy the Vampire Slayer, The X-Files et Supernatural: des séries centrales}

5 Nombreuses sont les séries fantastiques qui pourraient servir à étudier l'imaginaire américain de la forêt; par souci de clarté cependant, le présent article accompagne les descriptions générales d'études de cas empruntés à trois séries particulièrement représentatives du paysage audiovisuel anglophone. Le réseau intertextuel qu'elles constituent, à la fois les unes par rapport aux autres (Borsellino 2009) et pour l'ensemble de la production télévisuelle fantastique étasunienne, a été bien étudié ces dernières années $^{3}$. Leurs intrigues structurent un imaginaire des États-Unis et, plus particulièrement, de son espace géographique et d'un monde naturel (Dubois, 2012), dont l'ouverture parfois inquiétante s'oppose au huis-clos policé et malsain de la banlieue résidentielle (Billard \& Brennetot, 2009).

Créée en 1997 par Joss Whedon et diffusée jusqu'en 2003, la série Buffy the Vampire Slayer évoque les aventures de Buffy Summers, adolescente puis jeune femme choisie par les Forces du Bien pour mener leur lutte contre les Forces du Mal, dans la petite ville de Sunnydale, en Californie. Si la fonction première de la Tueuse est, comme le titre du programme l'indique, de débarrasser la ville des vampires, Buffy croise au fil des épisodes une grande variété de monstres et de démons, qu'elle combat avec l'aide de ses amis. Stylistiquement, Buffy the Vampire Slayer se caractérise par la rencontre du teen drama (programme pour adolescents, décrivant la vie de ses héros dans un univers scolaire) et du horror show (programme d'horreur, mettant en scène des monstres effrayants), c'est-àdire par l'association du rythme soutenu de l'action surnaturelle, qui implique 
notamment des combats, et de la rupture de ton provoquée l'introduction d'éléments comiques. Historiquement, cette série constitue à ce jour le matériau le plus exploité par les études universitaires sur ce type de productions télévisuelles.

À peu près contemporaine de Buffy, la série The X-Files a été créée en 1993 par Chris Carter et diffusée par la Fox jusqu'en 2002. Elle raconte les tribulations de deux agents du F.B.I, Fox Mulder, brillant psychologue criminel mis au ban de l'institution à cause de son intérêt pour le paranormal, et Danna Scully, médecin légiste rationnelle. La série se partage en deux tendances. D'un côté, des épisodes isolés mettent en scène des histoires qui tiennent du surnaturel ou de la science-fiction, avec des thèmes originaux ou des créatures du bestiaire traditionnel et de l'autre, des arcs narratifs développent l'histoire de la lutte des deux agents pour triompher d'un complot gouvernemental destiné à dissimuler l'existence des extraterrestres.

8 Postérieure à ces deux programmes et en partie inspirée par eux, la série Supernatural, encore en production, est créée en 2005 par Eric Kripke. Elle est consacrée à deux frères, Sam et Dean Winchester, qui sillonnent en voiture les États-Unis pour chasser monstres et fantômes d'abord (Tosenberger 2010), puis pour contrecarrer les sombres desseins des Anges et des Démons, qui désirent s'affronter lors de l'Apocalypse. Reprenant d'abord les ressorts cinématographiques et narratifs du film d'horreur, la série retrouve après la première saison les codes alors bien installés de la série fantastique, auxquels s'ajoute la double influence du western et du road movie.

Ces trois séries partagent un bestiaire, des lieux, des codes et une structure actantielle (Dubois 2012), largement inspirés du folklore traditionnel ; cette communauté est du reste signalée par les références à $X$-Files dans Buffy et aux deux premières séries dans Supernatural. Elles figurent parmi les représentantes les plus remarquables d'un genre (ou, si l'on préfère, d'un domaine) aujourd'hui florissant où l'on peut encore compter, à titre d'exemples, des programmes tels que Sanctuary (Damian Kindler, 2008-2011), Warehouse 13 (Jane Espenson et D. Brent Mote, 2009-en cours), Angel (Joss Whedon et David Greenwalt, 1999-2004), Grimm (Stephen Carpenter, David Greenwalt et Jim Kouf, 2011-en cours) ou encore Fringe (J. J. Abrams, Alex Kurtzman et Roberto Orci, 2008-en cours). En d'autres termes, ces trois séries fondatrices sont tout à la fois les agents et les miroirs d'un imaginaire partagé, reproduit et diffusé, un imaginaire où les créatures fantastiques et monstrueuses sont loin d'être reléguées aux affabulations d'un temps révolu. À contrecourant de l'hypothèse de la télévision comme médium réaliste (Jost 2003), les séries fantastiques proposent une résolution alternative de problématiques contemporaines (Dubois 2013).

\section{La forêt et le monstre}

Le monstre sanguinaire est indubitablement l'unité fondamentale de la construction de cet imaginaire populaire de la forêt. Grizzli surnaturel, il est l'entité animale qui dévore les promeneurs imprudents, trop prompts à s'aventurer en dehors des sentiers battus. Dans Being Human (Toby Whithouse, 2008-en cours), c'est dans la forêt que s'éveille parfois le loup-garou après ses transformations, quand l'animalité de sa forme surnaturelle l'a emporté sur son humanité et l'a conduit à briser les chaînes par lesquelles il s'était contraint. $\mathrm{Nu}$, couché près du cadavre sanglant d'un animal, il prend ainsi conscience de l'altérité essentielle qui est la sienne. 
11 L'épisode «Phases » (saison 2, épisode 15) de Buffy the Vampire Slayer participe de la même construction du personnage lycanthropique. Après l'attaque sauvage de deux d'entre eux par une créature d'abord non-identifiée, Buffy et son groupe d'amis établissent un lien entre de nombreuses agressions attribuées à des animaux sauvages et les cas de lycanthropie documentés dans leur bibliothèque mystique : le loup-garou devient alors l'expression paroxystique d'une nature dangereuse, qui remet en cause la place dominante de l'être humain dans la chaîne alimentaire.

C'est alors dans la forêt que se déroule d'abord la traque du lycanthrope; Buffy, armée d'un fusil tranquillisant, rencontre lors de sa patrouille un chasseur qui s'est fait une spécialité du loup-garou et en exhibe les dents comme autant de trophées. Dans Supernatural, le lien entre la chasse aux bêtes sauvages et la traque des monstres est étendu à l'ensemble du bestiaire: quand Buffy est qualifiée de slayer («tueuse »), les frères Winchester se définissent comme des hunters (" chasseurs ») et tout le vocabulaire cynégétique se déploie ainsi dans la série télévisée, de la carabine aux pavillons de chasse. De la même façon, dans « Shapes » (The X-Files, saison 1, épisode 19), les attaques de bétail sont d'abord attribuées à un animal sauvage et, dans le ranch que visitent pour les besoins de leur enquête les agents Mulder et Scully, de nombreux trophées empaillés suspendus aux murs viennent rappeler le contexte cynégétique.

13 La partie animale du loup-garou l'emporte donc sur sa partie humaine et la représentation de sa chasse par le héros et, parfois, ses opposants, n'emprunte rien à celle de la chasse à l'homme que j'évoquais plus haut à propos de Dollhouse et dont Antonio Dominguez Leiva a récemment rappelé l'histoire cinématographique. Si la chasse à l'homme joue de l'humanité de la victime chassée, la chasse au loup-garou, elle, se consacre exclusivement à l'animalité de la proie elle-même chasseresse. Ce n'est qu'une fois le loup-garou identifié et capturé, incarné par un acteur humain que, dans Being Human comme dans Buffy, le paradoxe de sa nature est exploré par la trame narrative. Une fois le loup-garou $\mathrm{Oz}$ découvert par Buffy et ses amis, il est placé les nuits de pleine lune dans une salle de la bibliothèque, sous la surveillance du petit groupe : dans la ville et l'univers humain, le loup-garou est un humain dont l'essence a été altérée ; en forêt, c'est un animal sauvage surpuissant qu'il s'agit de chasser ou, tout du moins, de contenir.

Cet imaginaire ambigu du monstre de la forêt, dont le loup-garou est l'expression la plus familière, s'exprime au-delà des fictions proprement fantastiques. Dans la série Bones (Hart Hanson, 2005-en cours), au cours de "The Truth in the Myth" (saison 6, épisode 18), les deux enquêteurs parfaitement rationnels tentent de résoudre une affaire qui, selon les légendes locales, impliquerait el Chupacabra (littéralement, "le suceur de chèvres »), une créature mythique à mi-chemin entre le loup-garou et le vampire, également évoquée dans The X-Files. Plus généralement, lorsqu'un corps est découvert en forêt, le docteur Brennan, anthropologue judiciaire, s'attache à déterminer qui de la bête sauvage ou de l'être humain est responsable de la mort. La forêt est ici le décor qui favorise les explications les plus sauvages jusqu'à faire émerger, dans un univers semblable aux nôtres, réaliste, des causes surnaturelles.

Cette unité fondamentale de l'imaginaire forestier qu'est le monstre, loup-garou ou bête sauvage mythique, n'est plus l'occasion médiévale de la rencontre entre le héros et un autre monde; il est le générateur d'une opposition frontale où l'être humain et le monstre sont chacun des chasseurs chassés. Dans sa dimension cynégétique, la forêt est ainsi un lieu hostile, qui n'est plus l'autre de la ville dans un système qui formerait 
l'ensemble des lieux humains, mais l'autre de l'humanité, à proprement parler un autre monde, réglé par des lois différentes.

\section{La forêt et le presque-humain} doute l'un des plus clairs. Emprunté à la mythologie des Algonquiens, le wendigo est un esprit qui prend possession des êtres humains (parfois un humain qui se transforme en monstre) pour les forcer à dévorer leurs semblables. Ce matériau originel, assez proche de la lycanthropie et d'ailleurs traité comme tel dans « The Wendigo » (saison 1, épisode 12) de la série Charmed (Constance Burge, 1998-2006), est retravaillé par Supernatural afin d'insister sur la continuité qui existe entre l'être humain et la créature cannibale. Selon Dean Winchester, le wendigo est un être humain que l'isolement dans une petite communauté au sein de la forêt aurait conduit au cannibalisme. En s'adonnant à cette pratique, l'être humain est progressivement transformé et devient le chasseur parfait: rusé, rapide et silencieux, mais habité par un constant besoin de chair humaine. Figure traditionnelle du tabou, donc, que la série rationalise en lui associant une explication géographique. ce qui en conditionne l'apparition : c'est l'isolement qui crée la sauvagerie. Le wendigo représente un état intermédiaire entre les humains que l'isolement a rendu fous et auxquels, parfois, il a donné une apparence monstrueuse, et les créatures anthropomorphes qui évoluent depuis toujours au sein de la forêt.

première extrémité de ce spectre est représentée de manière similaire dans "The Benders » (Supernatural, saison 1, épisode 15) et «Home » (The X-Files, saison 4, épisode 2). Dans « The Benders », Sam Winchester est enlevé par une famille de chasseurs qui vivent reclus dans les bois et dont le seul plaisir est la chasse à l'homme. Le décor et les costumes des personnages sont destinés à rappeler au téléspectateur ceux des maisons hantées et de leurs fantômes, si fréquents dans le reste de la série. Dans « Home », les agents Mulder et Scully se trouvent aux prises avec une famille peuplée d'enfants consanguins, vivant isolée à l'orée des bois; la difformité des corps, conséquence de générations de consanguinité, interroge, aux yeux des deux héros, les limites de l'humanité. Dans un cas comme dans l'autre, la forêt est un espace qui sépare un petit groupe d'êtres humains du reste de la civilisation et éveille en eux des instincts inhumains, qui brisent les tabous fondamentaux de l'organisation sociale : l'inceste, l'homicide et le cannibalisme (The XFiles, « Our Town », saison 2, épisode 24).

À l'autre extrémité de ce spectre se trouvent les créatures que la forêt elle-même a engendrées. Parmi d'autres, «The Jersey Devil » (The X-Files, saison 1, épisode 5) évoque l'histoire d'une créature anthropomorphe, parente de l'être humain, qui évolue dans une forêt du New Jersey depuis des générations. Le recul de son habitat naturel la pousse à aller chercher, pour ses petits, de la nourriture dans les quartiers périphériques de la ville la plus proche et c'est ainsi qu'elle attaque les êtres humains. À ces espèces endémiques il 
faut ajouter celles qu'ont produit des siècles d'adaptation. Dans "Detour " (The X-Files, saison 5, épisode 4), Mulder et Scully découvrent que des conquistadors perdus dans la forêt ont pu donner naissance à une nouvelle espèce entre l'être humain et l'animal.

21 La forêt n'est donc pas seulement le refuge de bêtes sauvages fantastiques qui entretiennent avec l'humanité une relation duelle d'adversité essentielle; elle peut être également ce qui fait émerger de l'humanité elle-même quelque chose qui n'est plus tout à fait humain. Le caractère surnaturel des créatures évoquées, parfois mal déterminé (et c'est le propre de l'indécision fantastique), naît d'une rupture dans l'ordre hermétique des choses, c'est-à-dire dans l'organisation logique du monde qui maintient de part et d'autre d'une frontière fermement définie l'humain et l'animal. Avec le loup-garou, la forêt est l'espace déshumanisé et par conséquent irrationnel ; avec le wendigo, elle est l'espace déshumanisant.

\section{La forêt vivante}

Le motif de la forêt vivante constitue bien entendu l'aboutissement de cette construction imaginaire : refuge de la bête d'abord, source de la bestialité ensuite, la forêt peut devenir elle-même un organisme vivant ou, à plus strictement parler, un écosystème indépendant. La forêt est alors non seulement un espace mais un ensemble de créatures vivantes, incarnation polymorphe de la Nature, dans laquelle sont compris les insectes, les animaux non-anthropomorphes et les arbres eux-mêmes. Cette forêt est un monde à part qui rentre en collision avec le monde des êtres humains et la réaction violente de la forêt doit conduire le téléspectateur à s'interroger sur son rapport avec la nature.

Dans la nouvelle version de la série britannique à grand succès Doctor Who (Russell $\mathrm{T}$. Davies puis Steven Moffat, 2005-en cours), l'épisode "The Widow and the Wardrobe" (épisode spécial de Noël, 2011) raconte la manière dont les arbres d'une forêt située sur une planète lointaine capturent une mère et ses deux enfants pour échapper à l'incinération projetée par une grande compagnie énergétique. La contrainte, d'ailleurs fort légère, exercée par la forêt sur l'être humain est une réponse défensive à la menace d'extinction et c'est d'abord comme un univers de conte de fées, où les arbres en guise de fruits produisent des décorations de Noël, que la forêt est filmée. L'intensification de la violence ne modifie pas nécessairement cette disposition fondamentale. Dans le double épisode "Silence in the Library » et «Forest of the Dead » (saison 4, épisodes 8 et 9), les voyageurs temporels, en visite sur une planète-bibliothèque, sont attaqués par les Vashta Nerada, créatures de l'ombre vivant ordinairement dans les forêts, mais que la production massive de livres a conduites jusqu'à ce monde. L'adversité naît alors d'une incompréhension mutuelle entre le monde des êtres humains et le monde des êtres de la forêt, incompréhension dont le téléspectateur ne peut imputer la faute qu'aux techniques d'exploitation et de production industrielles.

L'argument est semblable dans « Darkness Falls » (The X-Files, saison 1, épisode 20), où un groupe de bûcherons employés par une grande entreprise vient à disparaître dans une forêt du nord des États-Unis. Les agents Mulder et Scully ne tardent pas à découvrir que les hommes ont été victimes d'un essaim d'insectes assez semblables aux Vashta Nerada de Doctor Who; l'espèce, jadis disparue, a été préservée dans les anneaux d'un arbre centenaire et libérée par l'action de la tronçonneuse. La chronologie de l'agression entre l'être humain et la nature est ici inverse à ce qu'elle était dans le cas du loup-garou : c'est l'être humain qui frappe d'abord et la forêt ne fait guère que se défendre. De la même 
façon, dans "Bugs" (Supernatural, saison 1, épisode 8), les habitants d'une banlieue fraîchement construite sont tués par les insectes de la forêt et le projet des promoteurs immobiliers est arrêté avant même de pouvoir atteindre son terme. Aux insectes sont parfois substitués les végétaux et dans "Field Trip » (The X-Files, saison 6, épisode 21), Mulder et Scully sont les victimes des hallucinations provoquées par d'immenses champignons, au cœur d'une forêt de la Caroline du Nord. Ces épisodes sont souvent l'occasion pour les héros principaux de la série de développer un message écologiste, sans doute fort conventionnel, en évoquant la puissance de la nature, la fragilité de l'être humain et l'outrecuidance de ceux qui prétendent pouvoir dominer ces éléments ancestraux.

Ces épisodes, qui ne répondent pas au schéma cynégétique traditionnel fondé sur la confrontation des héros à un monstre qu'il s'agit de chasser, que ce monstre soit ou non humain, mettent en évidence l'étrangeté constitutive qui fonde l'imaginaire américain de la forêt : si la forêt est la terre d'élection des monstres, si elle en produit elle-même, c'est qu'elle est intrinsèquement un autre monde, imperméable à l'activité humaine.

\section{Les forêts merveilleuses}

Il importe cependant de nuancer cette description. S'il est vrai que les séries proprement fantastiques et, parfois, les séries de science-fiction, tendent à offrir de l'espace forestier une représentation sombre, au sens propre comme au sens figuré, multipliant les scènes nocturnes qui obscurcissent le plan, comme dans True Blood (Alan Ball, 2008-en production) intensifiant la violence de la vie sauvage, cette impression est parfois tempérée par des productions qui reprennent plus volontiers le motif de la forêt merveilleuse, terre de créatures magiques bienveillantes.

Son expression la plus ténue est peut-être celle du refuge. Tout au long de la saison 6, les frères Winchester de la série Supernatural se cachent de leurs nouveaux ennemis dans les pavillons de chasse de la forêt. De la première série Robin Hood sur la BBC (Joy Harrington, 1953) à la récente série Robin Hood, toujours sur la BBC (Dominic Minghella et Foz Allan, 2006-2009), les séries consacrées au héros de la forêt de Sherwood offrent l'archétype de cette situation. De la même façon, c'est dans les cavernes au cœur de la forêt de Rugosa que le Grand Maître Jedi Yoda et ses commandos trouvent un refuge contre l'armée d'Assajj Ventress (Star Wars: The Clone Wars, saison 1, épisode 1); c'est dans une forêt encore que certains héros de la série anglaise The Fades (Jack Thorne, 2011) se protègent des fantômes. Cette situation n'est pas surprenante au regard de ce que nous venons de décrire à propos de la forêt vivante. La violence de la forêt vivante dénonce les exactions humaines et transforme la forêt en critère de la morale: seuls peuvent y survivre les héros vertueux, qui ne sont pas soumis aux technologies et aux systèmes de production du monde dans lequel ils sont nés.

De façon moins complexe, de nombreuses séries animées pour enfants explorent l'univers de la forêt merveilleuse et filment son bestiaire le plus traditionnel. Dans la série américaine Dora The Explorer (Chris Gifford, Valerie Walsh Veldes et Eric Weiner, 2000-en cours), la jeune exploratrice croise dans la forêt des licornes («Isa's Unicorn Flowers", saison 5, épisode 4), des hiboux parlants (« Dora's Enchanted Forest Adventure », saison 6, épisodes 8 à 10) ou encore des fées (« Dora Saves the Snow Princess", saison 5, épisode 2). De la même façon, dans la série canadienne Franklin (Paulette Bourgeois et Brenda Clark, 
1997-2007), la petite tortue Franklin vit avec ses parents et d'autres animaux dans une forêt merveilleuse, tout comme les Smurfs (Hanna-Barbera, 1981-1989). Dans la production américano-franco-canadienne The Care Bears (Linda Denham et Elena Kucharik, 1985-1988), c'est encore dans la Forêt des Sentiments que les Bisounours se distraient («The Forest of Misfortunes », épisode 5a).

Il n'en demeure pas moins que ces exemples sont en très grande majorité contenus dans le domaine spécifique des dessins animés pour (très) jeunes enfants. Ils exploitent l'univers merveilleux des contes et des fables, dont ils reprennent l'imaginaire géographique et animalier. Les dessins animés moins enfantins miment eux plus volontiers les codes des séries pour adultes. Il est alors symptomatique que la forêt merveilleuse et bénéfique appartienne à l'univers idéalisé de la fiction de jeunesse, c'està-dire à un mode de représentation non réaliste.

\section{Remarques finales}

La présente typologie ne doit pas donner l'impression qu'il est toujours possible, pour les séries télévisées du monde anglophone, de classer telle manifestation de l'imaginaire de la forêt dans l'une des trois catégories évoquées. Ces catégories sont certes opératoires et, dans bien des cas, on trouvera que l'événement surnaturel est le fait soit d'un monstre qui s'abrite dans la forêt, soit d'un être humain modifié par l'isolement dans la nature, soit de la forêt elle-même en tant qu'entité vivante. Cependant, il arrive souvent que ces catégories soient perméables; nous l'avons vu, si le wendigo de Charmed relève du monstre de la forêt, au même titre que le loup-garou de Buffy the Vampire Slayer, celui de Supernatural est beaucoup plus proche des hommes des bois de The X-Files. Comme bien souvent dans les narrations fantastiques, littéraires, cinématographiques ou télévisuelles, la question posée par le monde naturel est celle des limites de l'humanité auxquelles se superposent les limites de la rationalité. Ce qui est humain, c'est-à-dire ce qui appartient au monde de l'être humain, c'est ce qui est compréhensible ; la forêt au contraire, séparée de la ville et de la civilisation, défie les outils traditionnels du raisonnement. Il est ainsi remarquable que les réflexes des enquêteurs de la série policière réaliste Bones, face à un cadavre trouvé en forêt, soient d'abord les mêmes que ceux de Mulder et Scully dans The $X$-Files : il faut déterminer si la victime a été attaquée par une bête sauvage. On aurait tort de proposer toujours deux grilles d'analyse, l'une pour les séries réalistes, l'autre pour les séries fantastiques (et, à plus forte raison, de science-fiction), tant il est vrai qu'elles partagent un imaginaire commun traité avec des styles différents.

31 À cette dimension, si l'on peut dire, anthropologique, qui interroge les limites de l'humain et du rationnel, la représentation de la forêt dans la série américaine, qui se distingue en cela assez nettement des fictions britanniques, canadiennes ou australiennes évoquées ici, est également riche d'implications politiques. Nous l'avons vu, ce ne sont pas toujours des familles qui se trouvent isolées et transformées par leur isolement. Dans «Die Hande die Verletz " (The X-Files, saison 2, épisode 14) comme dans "Scarecrow " ( Supernatural, saison 1, épisode 11), ce sont les notables de petites villes entourées par la forêt qui, en s'adonnant à la sorcellerie, sont responsables des meurtres qui s'y déroulent. La forêt par laquelle les héros passent pour accéder à la ville ou dans laquelle se déroule la première scène de l'épisode, avant le générique, sert de code visuel qui, au téléspectateur familier de ce genre de programmes, signale l'insularité d'une 
communauté. Se lit dans ces épisodes le malaise de la small-town America (Tovar 2011), espace en marge de l'État fédéral et de sa puissance civilisatrice (Gatefin 2012).

L'imaginaire de la forêt est dense : il peut servir de cadre, de terreau pour tout un épisode, ou fonctionner simplement comme un code aisément interprétable par le téléspectateur. Il est en tout cas celui d'un monde naturel essentiellement étranger, que l'être humain ne peut visiter qu'à ses risques et périls et dans lequel ne peuvent survivre que ceux qui sont prêts à renier leur propre humanité.

\section{BIBLIOGRAPHIE}

Abott, Stacey et Lavery David (éd). TV Goes To Hell : An Unofficial Road Map To Supernatural. Toronto : ECW Press, 2011.

Billard, Gérald et Brennetot, Arnaud. « Le huis clos ou l'exaltation du localisme communautaire dans les séries américaines ». GRAAT 9 (2009) : web.

Borsellino, Mary. « Buffy the Vampire Slayer, Jo the Monster Killer : Supernatural's Excluded Heroines ». In the Hunt : Unauthorized Essays on Supernatural. Dallas : BenBella Books, 2009. 107-18.

Dominguez Leiva, Antonio. « De Zaroff à Panem, jalons du pouvoir cynégétique à l'écran ». Pop-enstock 2012 : http://popenstock.ca/dossier/article/de-zaroff-panem-jalons-du-pouvoircynegetique-lecran (consulté le 19 avril 2014).

Dubois, François-Ronan. « Le mythe herculéen dans trois séries américaines : Supernatural, Buffy the Vampire Slayer et The X-Files ». e-lla 5 (2012) : http://revues.univ-provence.fr/e-lla/ index427.html (consulté le 19 avril 2014).

Dubois, François-Ronan. « Fantastique, science-fiction et résolution individualiste des crises globales dans les séries télévisées étasuniennes de 1990 à nos jours ». Magazine de la communication de crise \& Sensible 21 (2013) : 18-33.

Gatefin, Éric. « La représentation de l'espace américain dans The West Wing ». TV/Series 2 (2012). Jost, François. La télévision du quotidien : entre réalité et fiction. Bruxelles : De Boeck \& Larcier, 2003. Lavery David et Wilcox Rhonda V. (éd). Slayage 6.1 (2006) : http://slayageonline.com/Numbers/ slayage21.htm (consulté le 19 avril 2014).

Saunders, Corinne. The Forest of Medieval Romance. Cambridge : D. S. Brewer, 1993.

Tosenberger, Catherine. « 'Kinda like the folklore of its day' : Supernatural, fairy tales, and ostension ». Transformative Works and Cultures 4 (2010) : http://journal.transformativeworks.org/ index.php/twc/article/view/174/156 (consulté le 19 avril 2014).

Tovar, Elisabeth. «Supernatural "small-town America" : errance hantée dans les vestiges de l'Amérique industrielle ». Métropolitiques 2011 : http://www.metropolitiques.eu/Supernaturalsmall-town-America.html (consulté le 19 avril 2014). 


\section{NOTES}

1. « lieu antique de rencontre entre les dieux et les mortels " (je traduis).

2. « lieu romanesque de la quête, de l'idylle et de l'aventure " (je traduis)

3. Voir, entre autres, le premier numéro du sixième volume de la revue Slayage ainsi que l'introduction de TV Goes To Hell par Stacey Abbott.

\section{AUTEUR}

\section{FRANÇOIS-RONAN DUBOIS}

Université Stendhal - Grenoble 3, UMR LIRE.

Agrégé de Lettres Modernes et doctorant contractuel en littérature française. Spécialiste des séries télévisées du domaine anglophone (30 Rock, Supernatural, The X-Files, Buffy the Vampire Slayer, Doctor Who, The Newsroom, Studio 60 on Sunset Strip) et de la méthodologie de leur étude. Auteur de plusieurs articles et communications en journées d'études et colloques internationaux dont « Le mythe herculéen dans trois séries américaines » (e-lla) et « La configuration du temps dans la série télévisée » (Lignes de Fuite). 